\title{
Massless fermions in spatially flat FLRW space-times
}

\author{
Ion I. Cotăescu ${ }^{\text {a }}$ \\ West University of Timişoara, V. Pârvan Ave. 4, 300223 Timişoara, Romania
}

Received: 5 December 2021 / Accepted: 10 January 2022 / Published online: 24 January 2022

(C) The Author(s) 2022

\begin{abstract}
The propagation of the packets of left-handed plane wave solutions of the massless Dirac equation is studied in spatially flat Friedmann-Lemaître-RobertsonWalker (FLRW) space-times. Assuming that the observations are performed in physical frames with Painlevé-Gullstrand coordinates, the current and energy-momentum tensor are derived, emphasising their severe decay and moderate dispersion during propagation. As the wave packets move along null geodesics, interesting effects are produced by the apparent or event horizons.
\end{abstract}

\section{Introduction}

The theory of cosmic neutrinos in curved backgrounds was developed focusing on neutrino oscillations in various scenarios and gravitational fields [1-12] including the Schwarzschild metric, where the influence of the gravitational lensing was studied [13-16]. As the flavor transitions cannot be separated from propagation, the effective method of the wave packets was considered for deriving physical results [17-28] but without paying special attention to the propagation in gravitational fields.

We recently studied photon wave packets in spatially flat Friedmann-Lemaître-Robertson-Walker (FLRW) space time, showing that their propagation has new sound features which cannot be observed in the flat space-times $[29,30]$. We showed that, in expanding FLRW space-times, an observer at rest in its proper physical frame with Painlevé-Gullstrand coordinates $[31,32]$ measures photon wave packets emphasising the severe decay of the maximum intensity and moderate dispersion. In the present paper, we would like to continue this investigation in the case of the left-handed massless Dirac fermions propagating in spatially flat FLRW space-time but without flavour couplings. For this reason, we speak here about massless fermions instead of neutrinos.

\footnotetext{
a e-mail: i.cotaescu@e-uvt.ro (corresponding author)
}

We apply here the same method as in the case of the Maxwell field, starting with the observation that the massless Dirac equation can be solved analytically in any spatially flat FLRW space-time, in either conformal or physical frames. However, in contrast with the flat case, the plane wave solutions must depend explicitly on the initial conditions because of the physical space coordinates which are no longer homogeneous as the co-moving ones. In addition, we must take into consideration that the detector measuring the wave packets selects the fermions emitted by a fixed remote source. In fact, the detector filters the fermion momenta along the source-observer direction with the help of a suitable projection operator determining the amplitude of the detected one-dimensional wave packet as well as the expectation values and dispersions of the measured observables.

The principal results obtained here are the current and energy-momentum tensor of the plane wave packets of massless fermions propagating in spatially flat FLRW spacetimes with scale factors $a(t)$. We find that the current and the energy-momentum tensor evolve as $a(t)^{-3}$ and $a(t)^{-4}$ respectively while the dispersion is proportional to $a(t)$. Moreover, we show that the apparent motion of these wave packets along null geodesics give rise to interesting horizon effects as in the case of the photon wave packets we studied recently [29,30].

We start in the Sect. 2 defining the conformal and physical local charts which have to be completed with the local nonholonomic frames we need for studying the Dirac equation. In the next section we construct the plane wave solutions in conformal frames where we can take over the results of the flat case completed with the explicit initial conditions. After quantisation, the operators of the current and energymomentum tensor are written in the physical frame where their expectation values can be interpreted. In Sect. 4 we define the one-particle wave packets showing how these may be detected and deriving explicitly the expectation values of the current and energy-momentum tensor in the one-particle state defining the wave packet. An example of isotropic wave 
packet is given in Sect. 5 where we study the propagation in the matter-dominated and de Sitter expanding universes emphasising the horizon effects. Finally, we present some concluding remarks.

We use the Planck units with $\hbar=c=G=1$.

\section{Frames}

The space-times in which we study the propagation of the massless fermions are the local-Minkowskian spatially flat FLRW space-times, $(M, a)$, with scale factors $a(t)$. We consider here the frames formed by local charts of coordinates $x^{\mu}(\alpha, \mu, \nu, \ldots=0,1,2,3)$ in which we must define nonholonomic orthonormal frames using tetrads.

The simplest local charts are the conformal Euclidean ones, $\left\{t_{c}, \mathbf{x}_{c}\right\}$, with the conformal time $t_{c}$ and the co-moving Cartesian space coordinates $\mathbf{x}_{c}=\left(x_{c}^{1}, x_{c}^{2}, x_{c}^{3}\right)$, having line elements of the form

$\mathrm{d} s^{2}=a\left(t_{c}\right)^{2}\left(\mathrm{~d} t_{c}^{2}-\mathrm{d} \mathbf{x}_{c} \cdot \mathrm{d} \mathbf{x}_{c}\right)$.

Note that these coordinates were proposed for the first time by Lemaître [34] in de Sitter's universe. The conformal coordinates are useful in applications but these are different from the physical coordinates of Painlevé-Gullstrand type [31,32], formed by the cosmic time $t$ and the physical space coordinates $\mathbf{x}=\left(x^{1}, x^{2}, x^{3}\right)$. These coordinates can be introduced by substituting

$t_{c}=\int \frac{\mathrm{d} t}{a(t)}, \quad x_{c}^{i}=\frac{x^{i}}{a(t)}, \quad i, j, k, \ldots=1,2,3$,

for deriving the new line elements

$\mathrm{d} s^{2}=\left(1-\frac{\dot{a}(t)^{2}}{a(t)^{2}} \mathbf{x}^{2}\right) \mathrm{d} t^{2}+2 \frac{\dot{a}(t)}{a(t)} \mathbf{x} \cdot \mathrm{d} \mathbf{x} \mathrm{d} t-\mathrm{d} \mathbf{x} \cdot \mathrm{d} \mathbf{x}$,

of the physical local charts $\{t, \mathbf{x}\}$ of these manifolds. The function $a(t)=a\left[t_{c}(t)\right]$ is the usual FLRW scale factor while

$\frac{\dot{a}(t)}{a(t)}=\frac{1}{a(t)} \frac{\mathrm{d} a(t)}{\mathrm{d} t}=\frac{1}{a\left(t_{c}\right)^{2}} \frac{\mathrm{d} a\left(t_{c}\right)}{\mathrm{d} t_{c}}=\frac{\dot{a}\left(t_{c}\right)}{a\left(t_{c}\right)^{2}}$,

is the Hubble function for which we do not use a special notation. The inverse transformation $\{t, \mathbf{x}\} \rightarrow\left\{t_{c}, \mathbf{x}_{c}\right\}$ is obvious

$t=\int a\left(t_{c}\right) \mathrm{d} t_{c}, \quad \mathbf{x}=a\left(t_{c}\right) \mathbf{x}_{c}$.

In cosmology one prefers the FLRW coordinates $\left\{t, \mathbf{x}_{c}\right\}$ with the well-known line element

$\mathrm{d} s^{2}=\mathrm{d} t^{2}-a(t)^{2} \mathrm{~d} \mathbf{x}_{c} \cdot \mathrm{d} \mathbf{x}_{c}$,

since the co-moving space coordinates comply with the homogeneity of the universes with flat space sections. It is remarkable that the invariants depending only on $a(t)$, namely the Ricci and Kretschmann scalars, have the same form in physical and FLRW coordinates. The difference is that only in physical local charts we can separate the peculiar velocity from the recessional one [33]. For this reason, in what follows we use mainly physical frames resorting to the conformal ones as an auxiliary tool when these offer technical advantages. The final results are given in both the physical and FLRW frames.

The physical local chart with the metric (3) plays the role of a proper frame of an observer staying at rest in the origin $O(\mathbf{x}=0)$ and evolving along the vector field $\partial_{t}$ which, in general, is not a Killing one. In this local chart, the observer has a dynamical apparent horizon of radius

$r_{a}(t)=\left|\frac{a(t)}{\dot{a}(t)}\right|$.

In addition, this may have an event horizon whose radius is the distance [38,39],

$r_{e}(t)=\int_{t}^{\infty} \mathrm{d} t^{\prime} \frac{a(t)}{a\left(t^{\prime}\right)}$,

from which a photon emitted at time $t$ never arrives at the origin. In general, these horizons are different evolving with different velocities [30]. The exception is the de Sitter spacetime whose apparent and event horizons coincide. In physical coordinates, we thus have the opportunity of analysing the physical effects due to the apparent horizons [29] which are hidden when one uses the co-moving space coordinates.

To write the Dirac equation, we need to fix the tetrad gauge giving the vector fields $e_{\hat{\alpha}}=e_{\hat{\alpha}}^{\mu} \partial_{\mu}$ defining the local orthogonal frames, and the 1-forms $\omega^{\hat{\alpha}}=\hat{e}_{\mu}^{\hat{\alpha}} \mathrm{d} x^{\mu}$ of the dual coframes (labelled by the local indices, $\hat{\mu}, \hat{v}, \ldots=0,1,2,3$ ). The metric tensor of $(M, a)$ can be expressed now as $g_{\mu \nu}=$ $\eta_{\alpha \hat{\beta}} \hat{e}_{\mu}^{\hat{\alpha}} \hat{e}_{\nu}^{\hat{\beta}}$ where $\eta=\operatorname{diag}(1,-1,-1,-1)$ is the Minkowski metric. Here we restrict ourselves to the Cartesian diagonal tetrad gauge defined by the vector fields

$e_{0}=\frac{1}{a\left(t_{c}\right)} \partial_{t_{c}}=\partial_{t}+\frac{\dot{a}(t)}{a(t)} x^{i} \partial_{x^{i}}$,
$e_{i}=\frac{1}{a\left(t_{c}\right)} \partial_{x_{c}^{i}}=\partial_{x^{i}}$,

and the corresponding dual 1-forms

$\omega^{0}=a\left(t_{c}\right) \mathrm{d} t_{c}=\mathrm{d} t$,

$\omega^{i}=a\left(t_{c}\right) \mathrm{d} x_{c}^{i}=d x^{i}-\frac{\dot{a}(t)}{a(t)} x^{i} \mathrm{~d} t$,

which preserve the global $S O(3)$ symmetry allowing us to use systematically the $S O(3)$ vectors. When we use the charts with spherical coordinates we keep the same Cartesian gauge.

We obtain thus the frames $\{x ; e\}$ formed by a local chart $\{x\}$ and the local orthogonal frame and co-frame given by the tetrads $e$ and $\hat{e}$, as defined by Eqs. (9) and (10), respectively. The co-moving space coordinates of these frames can be 
transformed by the $E(3)$ isometries formed by space translations and global $S O(3)$ rotations. Note that in physical frames the translations are time-dependent while the rotations retain a global symmetry [33].

The local frames are defined up to local gauge transformations of the Lorentz group. In order not to affect the physical meaning, the entire theory must be invariant under local gauge transformations, generalising thus the Lorentz covariance of special relativity [35]. In what follows we study the massless fermions in the frames $\{x ; e\}$ of the space-times $(M, a)$.

\section{Quantum field}

We consider first the massless Dirac fermions in conformal frames where the Dirac equation, $E_{D} \psi=0$, of the massless quantum field $\psi$ is conformally covariant. This means that the Dirac operator in the conformal frame $\left\{t_{c}, \mathbf{x}_{c} ; e\right\}$,

$E_{D}=\frac{1}{a\left(t_{c}\right)}\left[i \gamma^{0} \partial_{t_{c}}+i \gamma^{i} \partial_{x_{c}^{i}}+\frac{3 i}{2} \frac{\dot{a}\left(t_{c}\right)}{a\left(t_{c}\right)} \gamma^{0}\right]$,

expressed in terms of Dirac matrices $\gamma^{\hat{\alpha}}$ in the chiral representation (with diagonal $\gamma^{5}$ ), allows the substitution $\psi \rightarrow$ $a\left(t_{c}\right)^{-\frac{3}{2}} \hat{\psi}$ that leads to the simpler equation

$$
\left(i \gamma^{0} \partial_{t_{c}}+i \gamma^{i} \partial_{x_{c}^{i}}\right) \hat{\psi}=0
$$

which has the same form and solutions as in special relativity. The difference is that the space-time $(M, a)$ is no longer homogeneous as the Minkowski one, such that the general solution we look for must depend explicitly on the initial condition $x_{c 0}=\left(t_{c 0}, \mathbf{x}_{c 0}\right)$, showing when and where this was prepared.

Under such circumstances, we may take over the results known in the flat case writing down the general form of the left-handed quantum field prepared at the time $t_{c 0}$ in the space point $\mathbf{x}_{c 0}$ as

$$
\begin{aligned}
\Psi\left(x_{c}\right) & \equiv \Psi_{L}\left(x_{c}\right) \\
& =\int \mathrm{d}^{3} p \sum_{\lambda}\left[U_{\mathbf{p}, \lambda}\left(x_{c}\right) \mathfrak{a}(\mathbf{p}, \lambda)+V_{\mathbf{p}, \lambda}\left(x_{c}\right) \mathfrak{b}^{\dagger}(\mathbf{p}, \lambda)\right],
\end{aligned}
$$

in terms of field operators, $\mathfrak{a}$ and $\mathfrak{b}$, and left-handed fundamental spinors,

$$
\begin{aligned}
U_{\mathbf{p}, \lambda}\left(x_{c}\right) & =\frac{\frac{1}{2}-\lambda}{\left[2 \pi a\left(t_{c}\right)\right]^{\frac{3}{2}}}\left(\begin{array}{c}
\xi_{\lambda}(\mathbf{p}) \\
0
\end{array}\right) e^{-i p\left(t_{c}-t_{c 0}\right)+i \mathbf{p} \cdot\left(\mathbf{x}_{c}-\mathbf{x}_{c 0}\right)} \\
V_{\mathbf{p}, \lambda}\left(x_{c}\right) & =\frac{\frac{1}{2}+\lambda}{\left[2 \pi a\left(t_{c}\right)\right]^{\frac{3}{2}}}\left(\begin{array}{c}
\eta_{\lambda}(\mathbf{p}) \\
0
\end{array}\right) e^{i p\left(t_{c}-t_{c 0}\right)-i \mathbf{p} \cdot\left(\mathbf{x}_{c}-\mathbf{x}_{c 0}\right)}
\end{aligned}
$$

which depend on the initial condition. The Pauli spinors of helicities $\lambda= \pm \frac{1}{2}$, related as $\eta_{\lambda}(\mathbf{p})=i \sigma_{2}\left[\xi_{\lambda}(\mathbf{p})\right]^{*}$, form an orthonormal system, $\xi_{\lambda}^{+} \xi_{\lambda^{\prime}}=\eta_{\lambda}^{+} \eta_{\lambda^{\prime}}=\delta_{\lambda, \lambda^{\prime}}$, and satisfy the eigenvalues problems

$\boldsymbol{\sigma} \cdot \mathbf{p} \xi_{\lambda}(\mathbf{p})=2 p \lambda \xi_{\lambda}(\mathbf{p})$,

$\boldsymbol{\sigma} \cdot \mathbf{p} \eta_{\lambda}(\mathbf{p})=-2 p \lambda \eta_{\lambda}(\mathbf{p})$,

that allowed us to derive the forms (14) and (15).

We now understand that the fundamental spinors of positive frequencies, $U$, are non-vanishing only for $\lambda=-\frac{1}{2}$ while those of negative frequencies, $V$, have only $\lambda=\frac{1}{2}$, as in the flat Minkowski space-time. For this reason we denote simply

$U_{\mathbf{p}}=U_{\mathbf{p}, \lambda=-\frac{1}{2}}, \quad V_{\mathbf{p}}=V_{\mathbf{p}, \lambda=\frac{1}{2}}$

the orthonormal solutions that satisfy

$$
\begin{aligned}
& \left(U_{\mathbf{p}}, U_{\mathbf{p}^{\prime}}\right)=\left(V_{\mathbf{p}}, V_{\mathbf{p}^{\prime}}\right)=\delta^{3}\left(\mathbf{p}-\mathbf{p}^{\prime}\right) \\
& \left(U_{\mathbf{p}}, V_{\mathbf{p}^{\prime}}\right)=\left(V_{\mathbf{p}}, U_{\mathbf{p}^{\prime}}\right)=0
\end{aligned}
$$

and form a complete system with respect to the Hermitian form,

$\left(\psi, \psi^{\prime}\right)=\int \mathrm{d}^{3} x_{c} a\left(t_{c}\right)^{3} \bar{\psi}\left(x_{c}\right) \gamma^{0} \psi^{\prime}\left(x_{c}\right)$,

where $\bar{\psi}=\psi^{+} \gamma^{0}$ is the Dirac adjoint of $\psi$.

Similarly, we denote the particle, $\mathfrak{a}(\mathbf{p})=\mathfrak{a}\left(\mathbf{p}, \lambda=-\frac{1}{2}\right)$, and antiparticle, $\mathfrak{b}(\mathbf{p})=\mathfrak{b}\left(\mathbf{p}, \lambda=\frac{1}{2}\right)$, field operators whose non-vanishing anti-commutation rules read

$$
\left\{\mathfrak{a}(\mathbf{p}), \mathfrak{a}^{\dagger}\left(\mathbf{p}^{\prime}\right)\right\}=\left\{\mathfrak{b}(\mathbf{p}), \mathfrak{b}^{\dagger}\left(\mathbf{p}^{\prime}\right)\right\}=\delta^{3}\left(\mathbf{p}-\mathbf{p}^{\prime}\right) .
$$

With these operators we may construct the momentum basis of the Fock space with an unique vacuum state, $|0\rangle$, defined as

$\mathfrak{a}(\mathbf{p})|0\rangle=\mathfrak{b}(\mathbf{p})|0\rangle, \quad\langle 0| \mathfrak{a}^{\dagger}(\mathbf{p})=\langle 0| \mathfrak{b}^{\dagger}(\mathbf{p})=0$.

just as in the flat case. Obviously, this is a generalised basis whose vectors having infinite norms do not represent physical states but allow one to construct such states as suitable linear combinations, i.e. the wave packets we have to study here.

The observed quantities are the expectation values of conserved or time-dependent operators. The conserved operators are the one-particle operators corresponding to the conserved quantities via the Noether theorem, namely the charge, momentum and angular momentum operators. In what follows we use only the momentum operator whose components can be written as

$$
\begin{aligned}
\mathscr{P}^{i} & =:\left(\Psi, \hat{P}^{i} \Psi\right): \\
& =\int \mathrm{d}^{3} p p^{i}\left[\mathfrak{a}^{\dagger}(\mathbf{p}) \mathfrak{a}(\mathbf{p})+\mathfrak{b}^{\dagger}(\mathbf{p}) \mathfrak{b}(\mathbf{p})\right],
\end{aligned}
$$


respecting the normal ordering of the operator products [36]. The notation $\hat{P}^{i}$ stands for the components of the orbital momentum operator which act as

$$
\left(\hat{P}^{i} \Psi\right)\left(x_{c}\right)=-i \partial_{x_{c}^{i}} \Psi\left(x_{c}\right),
$$

in the conformal frame where the co-moving space coordinates can be translated as in the flat case. We remind the reader that the corresponding isometries in physical frames are depending on time [33].

The expectation values of the time-dependent operators must be derived in physical frames, $\{t, \mathbf{x} ; e\}$, where these have physical meaning. We consider here the Dirac current (density) having the components

$$
\mathscr{J}^{\mu}(x)=: \bar{\Psi}(x) \gamma^{\mu}(x) \Psi(x), \quad \gamma^{\mu}(x)=e_{\hat{\alpha}}^{\mu}(x) \gamma^{\hat{\alpha}},
$$

and the energy-momentum tensor with symmetrised components [37],

$$
\begin{aligned}
\mathscr{T}^{\mu \nu}(x)= & \mathscr{T}^{v \mu}(x) \\
= & \frac{i}{2}:\left(\bar{\Psi}(x) \gamma^{(\mu}(x) \nabla^{v)} \Psi(x)\right. \\
& \left.-\overline{\nabla^{(v} \Psi}(x) \gamma^{\mu)}(x) \Psi(x)\right) .
\end{aligned}
$$

These operators are Hermitian conserved currents in the sense that their divergences are vanishing giving rise to the mentioned conserved one-particle operators [36].

\section{Detecting wave packets}

In what follows we study the propagation of the plane wave packets generated by one-particle physical states,

$|\alpha\rangle=\int \mathrm{d}^{3} p \alpha(\mathbf{p}) \mathfrak{a}^{\dagger}(\mathbf{p})|0\rangle$,

defined by square integrable functions, $\alpha(\mathbf{p})$, which must satisfy the normalisation condition,

$\langle\alpha \mid \alpha\rangle=\int \mathrm{d}^{3} p|\alpha(\mathbf{p})|^{2}=1$.

The corresponding 'wave function'

$\psi_{\alpha}\left(x_{c}\right)=\left\langle 0\left|\Psi\left(x_{c}\right)\right| \alpha\right\rangle=\int \mathrm{d}^{3} p U_{\mathbf{p}}\left(x_{c}\right) \alpha(\mathbf{p})$,

defines the normalised wave packet, $\left(\psi_{\alpha}, \psi_{\alpha}\right)=1$, in the conformal frame. In this approach, the expectation values of the one-particle operators in the state $|\alpha\rangle$ can be calculated simply by using the corresponding orbital operators. For example, the expectation values of the components of the momentum operator can be derived as

$\left\langle\alpha\left|\mathscr{P}^{i}\right| \alpha\right\rangle=\left(\psi_{\alpha}, \hat{P}^{i} \psi_{\alpha}\right)=\int \mathrm{d}^{3} p p^{i}|\alpha(\mathbf{p})|^{2}$, by using the Hermitian form (20) and the operator (24) in the conformal frame.

Let us imagine now that an observer, staying at rest in the origin $O$ of its proper frame with physical coordinates, $\{t, \mathbf{x} ; e\}_{O}$, measures a beam of massless fermions emitted at the initial time $t_{0}$ by a source $S$ situated in the space point $\mathbf{x}_{0}=-\mathbf{n} d a\left(t_{0}\right)$. These fermions propagate along the unit vector $\mathbf{n}$ until they are detected at the final time $t_{f}$ in the observer's origin. The time-independent parameter $d$ is the co-moving distance $S O$ while the physical distance evolves in time as $d(t)=\mathrm{d} a(t)[30,33]$. The fermion beam, described in the conformal frame $\left\{t_{c}, \mathbf{x}_{c} ; e\right\}_{O}$ by the wave packet (29), is detected in the origin $(\mathbf{x}=0)$ of the physical frame of the same observer, $\{t, \mathbf{x} ; e\}_{O}$, at the final time $t_{f}$. In this layout, the conformal and physical frames have Cartesian axes parallel to those of the orthogonal frame defined by the tetrads (9) and (10). We simplify the geometry by choosing these axes parallel with the orthonormal frame $\left\{\mathbf{e}_{1}, \mathbf{e}_{2}, \mathbf{e}_{3}\right\}$ such that $\mathbf{n}=\mathbf{e}_{3}$ gives now the direction $S O$.

The wave packet evolves causally until the detector of the observer $O$ measures some of its parameters selecting only the fermions coming from the source $S$ whose momenta are parallel with $\mathbf{e}_{3}$. This means that the domain of the momenta measured by $O$ is $[29,30]$

$\Delta=\left\{\mathbf{p} \mid-\frac{\Delta p}{2} \leq p^{1} \leq \frac{\Delta p}{2},-\frac{\Delta p}{2} \leq p^{2} \leq \frac{\Delta p}{2}, p^{3}>0\right\}$,

where $\Delta p$ is a small quantity. Therefore, the measured state $|\hat{\alpha}\rangle$, is given now by the corresponding projection operator $\Lambda_{\Delta}$ acting as

$|\hat{\alpha}\rangle=\Lambda_{\Delta}|\alpha\rangle=\int_{\Delta} \mathrm{d}^{3} p \alpha(\mathbf{p}) \mathfrak{a}^{\dagger}(\mathbf{p})|0\rangle$.

Moreover, we may evaluate the integrals over $\Delta$ as

$$
\begin{aligned}
\int_{\Delta} \mathrm{d}^{3} p F(\mathbf{p}) & =\int_{-\frac{\Delta p}{2}}^{\frac{\Delta p}{2}} \mathrm{~d} p^{1} \int_{-\frac{\Delta p}{2}}^{\frac{\Delta p}{2}} \mathrm{~d} p^{2} \int_{0}^{\infty} \mathrm{d} p^{3} F\left(p^{1}, p^{2}, p^{3}\right) \\
& \simeq(\Delta p)^{2} \int_{0}^{\infty} \mathrm{d} p F(0,0, p)
\end{aligned}
$$

according to the mean value theorem. Thus we can calculate the quantity

$\left\langle\alpha\left|\Lambda_{\Delta}\right| \alpha\right\rangle=\int_{\Delta} \mathrm{d}^{3} p \alpha(\mathbf{p})^{2}=(\Delta p)^{2} \kappa$,

where

$\kappa=\int_{0}^{\infty} \mathrm{d} p|\alpha(0,0, p)|^{2}$.

Under such circumstances, the new wave packet

$\left\langle 0\left|\Lambda_{\Delta} \Psi\left(x_{c}\right)\right| \alpha\right\rangle=\int_{\Delta} \mathrm{d}^{3} p U_{\mathbf{p}}\left(x_{c}\right) \alpha(\mathbf{p})$, 
behaves as a one-dimensional packet,

$\psi_{\hat{\alpha}}\left(x_{c}\right)=\int_{0}^{\infty} \mathrm{d} p \hat{U}_{p}\left(x_{c}\right) \hat{\alpha}(p)$,

depending on the new normalised wave function

$\hat{\alpha}(p)=\frac{\alpha(0,0, p)}{\sqrt{\kappa}} \rightarrow \int_{0}^{\infty} \mathrm{d} p|\hat{\alpha}(p)|^{2}=1$.

The momentum direction is now along the third axis which means that the polarisation $-\frac{1}{2}$ is just the spin projection on this axis. Therefore, we have $\xi_{-\frac{1}{2}}(\mathbf{p})=(0,1)^{T}$ obtaining the one-dimensional spinors,

$\hat{U}_{p}\left(x_{c}\right)=\frac{1}{a\left(t_{c}\right)^{\frac{3}{2}}} \frac{e^{-i p X_{c}}}{\sqrt{2 \pi}} \Xi, \quad \Xi=\left(\begin{array}{l}0 \\ 1 \\ 0 \\ 0\end{array}\right)$,

which depend on the variable

$X_{c}=t_{c}-t_{c 0}-x_{c}^{3}-d$,

corresponding to the initial point $\mathbf{x}_{c 0}=(0,0,-d)$ at the initial time $t_{c 0}$. With these preparations we can write the definitive form of the one-dimensional wave packet in the conformal frame,

$\psi_{\hat{\alpha}}\left(t_{c}, x_{c}^{3}\right)=\frac{1}{a\left(t_{c}\right)^{\frac{3}{2}}} \Phi\left(X_{c}\right) \Xi$,

where the function

$\Phi(X)=\frac{1}{\sqrt{2 \pi}} \int_{0}^{\infty} \mathrm{d} p e^{-i p X} \hat{\alpha}(p)$,

plays the role of an amplitude which encapsulates the principal integral we have to solve to study the propagation.

The observer $O$ can measure the parameters of the Dirac field in its proper physical frame, $\{t, \mathbf{x} ; e\}_{O}$, where the onedimensional wave packet,

$\psi_{\hat{\alpha}}\left(t, x^{3}\right)=\frac{1}{a(t)^{\frac{3}{2}}} \Phi(X) \Xi$,

depends on the new variable

$X=\int_{t_{0}}^{t} \frac{\mathrm{d} t^{\prime}}{a\left(t^{\prime}\right)}-\frac{x^{3}}{a(t)}-d$,

obtained after performing the substitution (2) in Eq. (40). In this frame, the wave packet is prepared in the physical space point $\mathbf{x}_{0}=\left(0,0,-\mathrm{d} a\left(t_{0}\right)\right)$ at time $t_{0}\left(\right.$ such that $\left.t_{c}\left(t_{0}\right)=t_{c 0}\right)$. Finally, this packet must be detected in $\mathbf{x}=0$ at the final time $t_{f}$ which is not yet derived.

To study the propagation of this wave packet, we focus on the expectation values of the operators (25) and (26) in the state $|\hat{\alpha}\rangle$. We calculate first the expectation values of the components (25) of the Dirac current using the wave packet (43) and the matrices (A.2). We obtain thus

$$
J^{\mu}=\left\langle\hat{\alpha}\left|\mathscr{J}^{\mu}(x)\right| \hat{\alpha}\right\rangle=\bar{\psi}_{\hat{\alpha}}(x) \gamma^{\mu}(x) \psi_{\hat{\alpha}}(x)=J V^{\mu},
$$

where

$J(t, X)=\frac{1}{a(t)^{3}}|\Phi(X)|^{2}$,

is the density while $V^{\mu}=\hat{V}^{\mu}+\bar{V}^{\mu}$ is the four-velocity in the physical frame where this can be split, in peculiar,

$$
\left(\hat{V}^{\mu}\right)=(1,0,0,1)
$$

and recessional,

$$
\left(\bar{V}^{\mu}\right)=\left(0, \frac{\dot{a}}{a} x^{1}, \frac{\dot{a}}{a} x^{2}, \frac{\dot{a}}{a} x^{3}\right),
$$

four-velocities [33]. As expected, the four-velocity is a null vector, $g_{\mu \nu} V^{\mu} V^{\nu}=0$, but it is remarkable that after separating the recessional part we remain with a flat null peculiar velocity which satisfies the null condition $\eta_{\mu \nu} \hat{V}^{\mu} \hat{V}^{\nu}=0$ with respect to the Minkowski metric $\eta$, as in special relativity. As mentioned before, this separation cannot be done in frames with FLRW coordinates where the current (45) takes the form $J_{c}=J V_{c}$, depending on the density (46), but the co-moving four-velocity

$V_{c}=\left(1,0,0, \frac{1}{a}\right)$.

Similarly, we derive the expectation values of the symmetrised components (26) of the energy-momentum tensor,

$$
\begin{aligned}
T^{\mu \nu}= & \left\langle\hat{\alpha}\left|\mathscr{T}^{\mu \nu}(x)\right| \hat{\alpha}\right\rangle=\frac{i}{2}\left(\bar{\psi}_{\hat{\alpha}}(x) \gamma^{(\mu}(x) \nabla^{\nu)} \psi_{\hat{\alpha}}(x)\right. \\
& \left.-\overline{\nabla^{(v} \psi_{\hat{\alpha}}}(x) \gamma^{\mu)}(x) \psi_{\hat{\alpha}}(x)\right)=I V^{\mu} V^{\nu},
\end{aligned}
$$

where the quantity

$I(t, X)=\frac{1}{a(t)^{4}} \frac{i}{2}\left(\Phi(X)^{*} \partial_{X} \Phi(X)-\Phi(X) \partial_{X} \Phi(X)^{*}\right)$,

will be called here the intensity since this is just the density of energy, $I=T^{00}$. Performing the same calculation in the FLRW frame we find that the components of the energymomentum tensor, $T_{c}^{\mu \nu}=I V_{c}^{\mu} V_{c}^{v}$, have the same intensity but depend on the co-moving velocity $V_{c}$ instead of $V$.

The density $J$ depends on the amplitude (42) which has the property $|\Phi(X)|<\Phi(0)$ whether $\hat{\alpha}$ is a test function. This means that, at any time $t$, the function (25) has an absolute (or global) maximum, $J(t, 0)$, for $X=0$ we call the maximum density. The existence of a corresponding maximum intensity is not obvious but if we chose a real valued test function $\hat{\alpha}$ then the quantity

$\Theta(X)=i \Phi(X)^{*} \partial_{X} \Phi(X)=\Phi(-X) \Phi_{1}(X)$, 
depends on the new function

$\Phi_{1}(X)=\frac{1}{\sqrt{2 \pi}} \int_{0}^{\infty} \mathrm{d} p p e^{-i p X} \hat{\alpha}(p)$,

which has the desired property $\left|\Phi_{1}(X)\right|<\Phi_{1}(0)$. Therefore, representing the intensity as

$I(t, X)=\frac{1}{a(t)^{4}} \frac{1}{2}(\Theta(X)+\Theta(-X))<\frac{\Theta(0)}{a(t)^{4}}=I(t, 0)$,

we understand that the maximum intensity, $I(t, 0)$, is obtained once again for $X=0$.

These maximum values are located at time $t$ in point $\left(0,0, x_{m}^{3}(t)\right)$ where

$x_{m}^{3}(t)=a(t)\left[\int_{t_{0}}^{t} \frac{\mathrm{d} t^{\prime}}{a\left(t^{\prime}\right)}-d\right]$,

solves the equation $X=0$. Remarkably, this is just the null geodesic passing through the point $S$ at the initial time $t_{0}$, when $x_{m}^{3}\left(t_{0}\right)=-d\left(t_{0}\right)=-d a\left(t_{0}\right)$, and arriving in $O$ at the final time, $t_{f}$, that can be derived by solving the equation

$x_{m}^{3}\left(t_{f}\right)=0 \rightarrow \int_{t_{0}}^{t_{f}} \frac{\mathrm{d} t}{a(t)}=d$,

resulting from the geodesic (55). The maximum density and intensity propagate along the null geodesic $S O$ along the third axis, in the domain $x_{m} \in\left[-d\left(t_{0}\right), 0\right]$, with the velocity

$v_{m}^{3}(t)=1+\frac{\dot{a}(t)}{a(t)} x_{m}^{3}(t)$

Therefore, the initial velocity

$v_{m}^{3}\left(t_{0}\right)=1-\dot{a}\left(t_{0}\right) d=1-\frac{d\left(t_{0}\right)}{r_{a}\left(t_{0}\right)}$,

is apparently different from the speed of light, but finally, at the time $t_{f}$ when the wave packet is detected, the observer $O$ measures just the speed of light, $v_{m}^{3}\left(t_{f}\right)=1$.

In expanding space-times $(M, a)$ with positive Hubble functions, the dragging back effect of the background determines initial velocities less than the speed of light. If at the initial time $t_{0}$ the source is inside the apparent horizon, then the geodesic motion is apparently accelerated from $0<v_{m}^{3}\left(t_{0}\right)<1$ up to $v_{m}^{3}\left(t_{f}\right)=1$. Moreover, when at the initial time the source is behind the apparent horizon, $d\left(t_{0}\right)>r_{a}\left(t_{0}\right)$, then $v_{m}^{3}\left(t_{0}\right)<0$ which means that the motion starts in the backward direction, stops and turns back to the forward direction accelerating to the detector which finally records the speed of light. In these space-times the maximum density follows the null geodesic having a decay $\propto a(t)^{-3}$ resulting from Eq. (46). The maximum intensity has the same trajectory with the difference that this has the decay $\propto a(t)^{-4}$ as in the case of the photons propagating in the same backgrounds $[29,30]$.

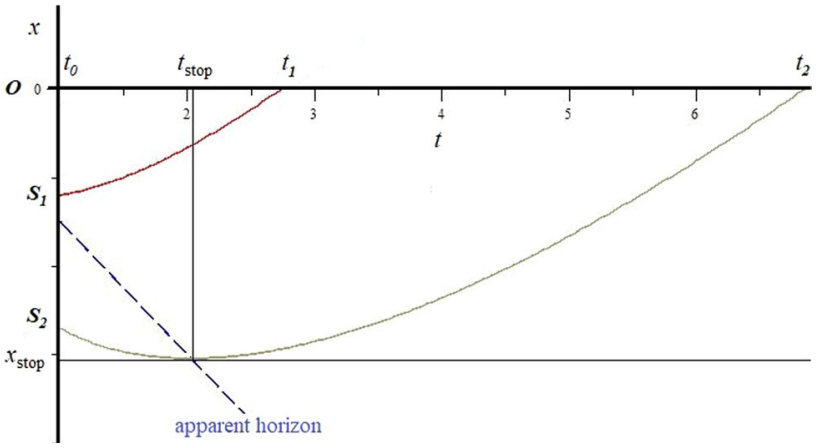

Fig. 1 The null geodesics of the maximum quantities of two fermions emitted simultaneously at the initial instant $t=t_{0}$. The first one is emitted by the source $S_{1}$ inside the dynamical event horizon while the second one is emitted by the source $S_{2}$ outside it. The second fermion starts in the backward direction, stops at the time $t_{\text {stop }}$ when it restarts in the forward direction accelerating until arriving in $O$ at the time $t_{2}>t_{1}$

It remains to evaluate the space dispersion of the wave packet, $\delta x(t)$, defined as the width of the function $I(t, X)$ at a given time. This depends on the width $\delta X$ of the function $\Theta(X)+\Theta(-X)$, which is a constant quantity depending on the profile of this function. Then, according to Eq. (44), we deduce that the physical dispersion, $\delta x(t)=\delta X a(t)$, evolves from $\delta x\left(t_{0}\right)=\delta X a\left(t_{0}\right)$ up to $\delta x\left(t_{f}\right)=\delta X a\left(t_{f}\right)$.

In conclusion, we may say that in expanding space-times, the apparent motion of the maximum quantities under consideration is accelerated with a severe decay and moderate dispersion, laying out specific horizon effects.

\section{Isotropic wave packet}

Let us illustrate now all the aforementioned effects in a brief graphical analysis studying the apparent motions of the wave packets of massless fermions in the proper physical frame $\{t, \mathbf{x} ; e\}_{O}$ of an observer $O$. In order to obtain intuitive profiles of the densities and intensities of these packets, we use arbitrary units, avoiding extremely large or small numbers.

We consider a simple isotropic wave packet which may be defined in spherical coordinates, $\mathbf{k}=(k, \theta, \phi)$, of the momentum space. We assume that at the initial time $t_{0}$ the source $S$ prepares the wave packet in $x_{0}=-d\left(t_{0}\right)$ defining the real-valued wave function in momentum representation as

$\alpha(p)=\frac{(2 \gamma)^{\gamma \bar{p}}}{2 \sqrt{\pi \Gamma(2 \gamma \bar{p})}} p^{\gamma \bar{p}-\frac{3}{2}} e^{-\gamma p}, \quad \gamma \bar{p}>\frac{3}{2}$.

This function is normalised,

$\int \mathrm{d}^{3} p \alpha(p)^{2}=4 \pi \int_{0}^{\infty} \mathrm{d} p p^{2} \alpha(p)^{2}=1$, 
depending on the free parameter $\gamma$ and the expectation value of the radial momentum of the emitted fermion,

$P=4 \pi \int_{0}^{\infty} \mathrm{d} p p^{3} \alpha(p)^{2}=\bar{p}$,

whose dispersion can be derived simply as

$\operatorname{disp} P=4 \pi \int_{0}^{\infty} \mathrm{d} p p^{4} \alpha(p)^{2}-\bar{p}^{2}=\frac{\bar{p}}{2 \gamma}$.

The observer $O$ measures the one-dimensional wave packet whose wave function (38) is re-normalised by using the constant

$\kappa=\int_{0}^{\infty} \mathrm{d} p \alpha(p)^{2}=\frac{\gamma^{2}}{2 \pi(\gamma \bar{p}-1)(2 \gamma \bar{p}-1)}$,

such that this now reads

$\hat{\alpha}(p)=(2 \gamma)^{\gamma \bar{p}-1} \sqrt{\frac{(2 \gamma \bar{p}-2)(2 \gamma \bar{p}-1)}{\Gamma(2 \gamma \bar{p})}} p^{\gamma \bar{p}-1} e^{-\gamma p}$.

With its help, we derive the expectation value of the momentum along the third axis,

$P_{f}^{3}=\int_{0}^{\infty} \mathrm{d} p p \hat{\alpha}(p)^{2}=\bar{p}-\frac{1}{\gamma}$,

which has to be detected by $O$ at the instant $t=t_{f}$ in $x^{3}=0$ with the dispersion

$\operatorname{disp} P_{f}^{3}=\int_{0}^{\infty} \mathrm{d} p p^{2} \hat{\alpha}(p)^{2}-\bar{p}^{2}=\frac{\bar{p}}{2 \gamma}-\frac{1}{2 \gamma^{2}}$,

proportional with $P_{f}^{3}$.

The amplitude in the proper physical frame of the observer $O$ is given by Eq. (42) as

$$
\begin{aligned}
\Phi_{L}(X)= & 2^{\gamma \bar{p}} \sqrt{\frac{\gamma \bar{p}-1}{\pi \gamma(2 \gamma \bar{p}-1) \Gamma(2 \gamma \bar{p})}} \\
& \times \Gamma\left(\gamma \bar{p}+\frac{1}{2}\right)\left(\frac{\gamma}{\gamma+i X}\right)^{\gamma \bar{p}-\frac{1}{2}} .
\end{aligned}
$$

With this amplitude, the density (46) and intensity (51) become

$$
\begin{aligned}
& J_{L}(t, X)=\frac{1}{a(t)^{3}} J_{0}\left(\frac{\gamma^{2}}{\gamma^{2}+X^{2}}\right)^{\gamma \bar{p}-\frac{1}{2}}, \\
& T_{L}(t, X)=\frac{1}{a(t)^{4}} T_{0}\left(\frac{\gamma^{2}}{\gamma^{2}+X^{2}}\right)^{\gamma \bar{p}+\frac{1}{2}},
\end{aligned}
$$

where we denote

$T_{0}=\left(\bar{p}-\frac{1}{2 \gamma}\right) J_{0}=\frac{\Gamma\left(\gamma \bar{p}+\frac{1}{2}\right)}{\sqrt{\pi} \gamma^{2} \Gamma(\gamma \bar{p}-1)}$

We observe that our density and intensity are different powers of a genuine Cauchy-Lorentz distribution. Therefore, these quantities have different widths,

$\delta X_{J}=2 \gamma \sqrt{2^{\frac{2}{2 \gamma \bar{k}-1}}-1}, \quad \delta X_{T}=2 \gamma \sqrt{2^{\frac{2}{2 \gamma \bar{k}+1}}-1}$,

determining their dispersion.

Example I: Matter-dominated universe

Let us consider first a massless fermion in the space-time $\left(M, t^{\frac{2}{3}}\right)$ with the scale factor $a(t)=t^{\frac{2}{3}}$ which is known as the matter-dominated universe. The gravitational source producing this FLRW space-time is a perfect fluid whose density (of matter or energy) $\rho$ and pressure $p$ read

$\rho=\frac{1}{6 \pi t^{2}}, \quad p=0$.

The density has the Big Bang singularity at $t=0$, which means that the condition $t>0$ is mandatory. An observer at rest in the origin of its proper physical frame $\{t, \mathbf{x} ; e\}_{O}$ in $\left(M, t^{\frac{2}{3}}\right)$ has a dynamical apparent horizon on a sphere of radius $r_{a}(t)=\frac{3}{2} t$ expanding with the constant velocity $v_{a}=\frac{3}{2}$.

We assume that, at the initial moment $t_{0}$, two sources, $S_{1}$ situated inside the apparent horizon and $S_{2}$ situated outside, emit simultaneously two massless fermions in the same state defined by the wave function (59). These fermions follow the null geodesics plotted in Fig. 1 arriving to the observer $O$ at the final times resulting from Eq. (56), i. e, $t_{1}$ and $t_{2}$, respectively.

The fermion emitted by $S_{1}$ inside the apparent horizon starts with an initial velocity smaller than 1 , because of the dragging effect of the background, propagating then accelerated straightforward to $O$ as in Fig. 2 where we plot both the intensity (69) and density (68) in a logarithmic scale.

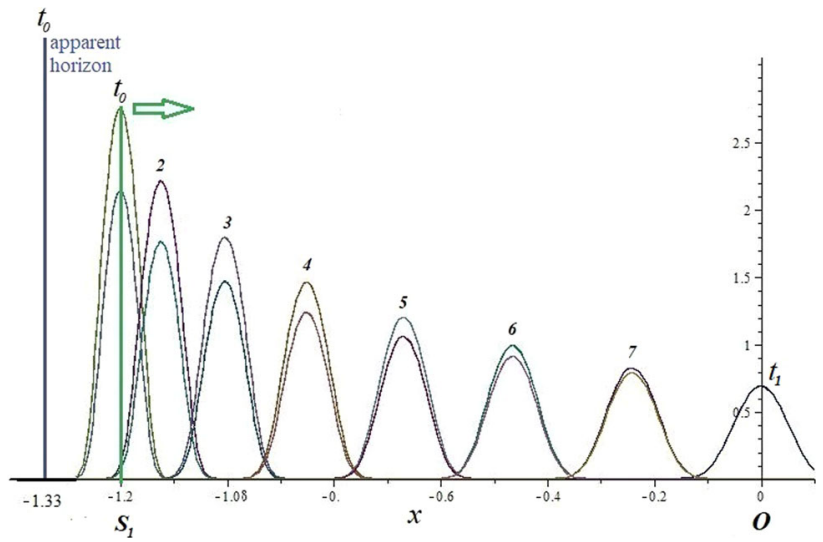

Fig. 2 The consecutive profiles in a logarithmic scale of the intensity and density (smaller) of the fermion emitted by the source $S_{1}$. This starts with an initial velocity less than one because of the dragging effect, and then it accelerates up to the speed of light measured in $O$. The decay and dispersion are obvious 


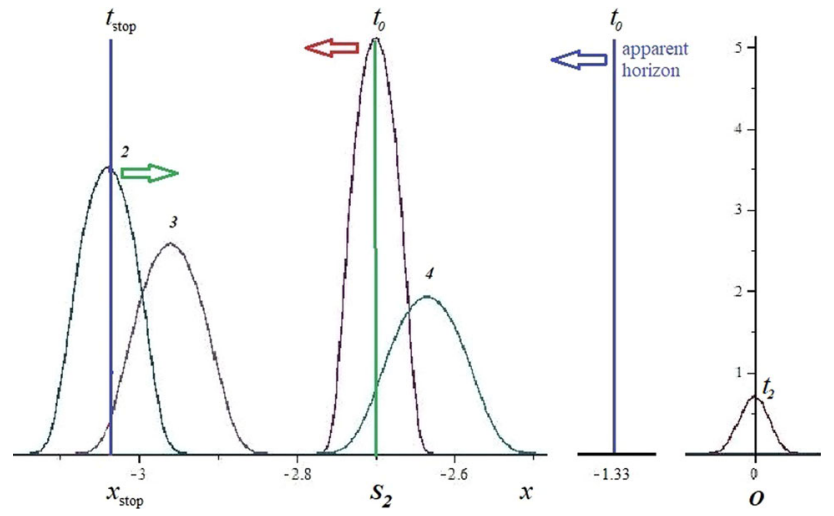

Fig. 3 The consecutive profiles in a logarithmic scale of the intensity of the fermion emitted by the source $S_{2}$ which starts in the backward direction turning back in $x_{\text {stop }}$ to the observer $O$ where this arrives at the instant $t_{2}$. The decay and dispersion are present during the entire propagation

In contrast, the fermion emitted by the source $S_{2}$ starts in the backward direction, with $v_{m}^{3}\left(t_{0}\right)<0$, because the dragging effect is stronger outside the event horizon. This fermion goes in the backward direction decelerating until it stops when the dynamical apparent horizon reaches it at instant $t_{\text {stop }}$ and distance $\left|x_{\text {stop }}\right|>d$. From this static position the fermion restarts in the forward direction approaching accelerated to the detector in $O$ which records the speed of light.

This remarkable effect produced by the apparent horizon can be pointed out exclusively in the physical frame where one uses the physical space coordinates. However, this apparent scenario, presented in Fig. 3, cannot be observed directly as the fermion moves causally during propagation, being measured/observed only by the detector in $O$. Nevertheless, indirect methods can be found for revealing this effect as in the photon case where the redshift magnitude may give us information about the relative position between source and dynamical horizon at the initial time [30].

\section{Example II: de Sitter expanding universe}

The second example is of a massless fermion in the expanding portion of the de Sitter space-time, known as the de Sitter expanding universe, $\left(M, a_{d S}\right)$, having the de Sitter scale factor $a_{d S}(t)=\exp \left(\omega_{d S} t\right)$, with $\omega_{d S}=\sqrt{\frac{\Lambda}{3}}$ and $t \in \mathbb{R}$. This manifold does not have a perfect fluid produced by the cosmological constant $\Lambda$ in vacuum. In this space-time the apparent horizon on the sphere of radius $r_{a}=\omega_{d S}^{-1}$ is in fact a static event horizon separating different domains of causality $[38,39]$. Therefore, the only fermions arriving in $O$ are emitted by sources inside the event horizon as in Fig. 4 where we plot the consecutive profiles of the intensity and density affected by decay and dispersion. If a similar wave packet is

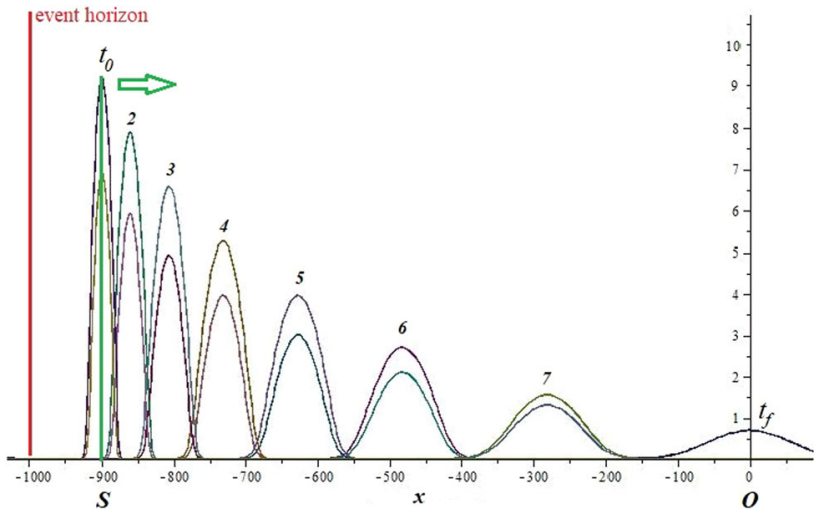

Fig. 4 The consecutive profiles in a logarithmic scale of intensity and density of a wave packet of a massless fermion emitted inside the event horizon. We observe the decay and dispersion

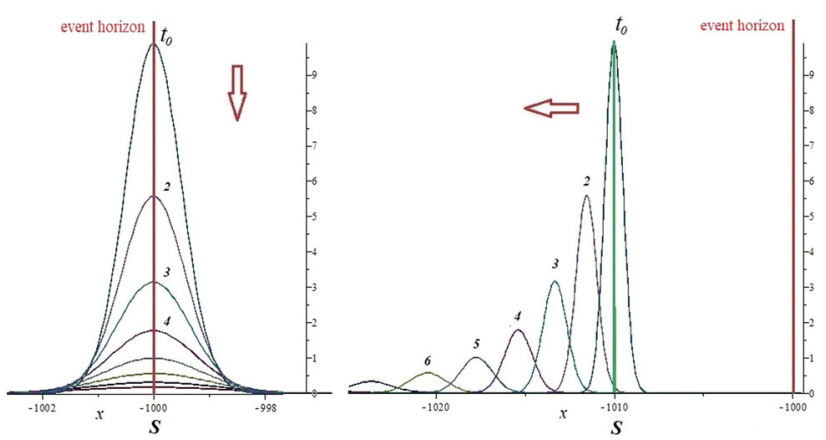

Fig. 5 Horizon effects produced by the event horizon of the de Sitter space-time which traps the packets prepared on it and reflects the packets prepared beyond it. Consecutive profiles of the intensity are plotted in both cases in a logarithmic scale

prepared just on the event horizon, then it collapses without moving as in the left panel of Fig. 5. The wave packets prepared outside the event horizon propagate in the backward direction as being reflected by this horizon. The evolution of the intensity in this case can be seen in the right panel of Fig. 5.

\section{Concluding remarks}

We described completely the propagation of the packets of left-handed plane waves of massless Dirac fermions as observed in the physical frames of the spatially flat FLRW space-times.

An observer may measure in its proper physical frame the expectation value of the fermion conserved momentum (65), the density (46) and the intensity (51). This gets thus the opportunity of estimating the emitted density and intensity,

$$
J_{\mathrm{emitt}}=J_{\mathrm{obs}} \frac{a\left(t_{f}\right)^{3}}{a\left(t_{0}\right)^{3}}, \quad I_{\mathrm{emitt}}=I_{\mathrm{obs}} \frac{a\left(t_{f}\right)^{4}}{a\left(t_{0}\right)^{4}},
$$


which depend only on the initial and final times. Unfortunately, the initial time cannot be determined directly as in the case of the light where the redshift gives the ratio of initial and final scale factors according to the Lemaître equation $[40,41]$ of Hubble's law $[42,43]$. Instead, one may deduce the initial time from other observations as, for example, detecting simultaneously fermions and photons from the same source.

In general, the energy is not a conserved quantity in spatially flat FLRW space-times except for the de Sitter expanding universe. Nevertheless, we can use the energy operator $\hat{H}=i \partial_{t}$ whose expectation values give the evolving energy which may be different from that defined in special relativity [44]. Moreover, this operator does not commute with the conserved momentum (23) that acts as $\hat{P}^{i}=-i a(t)^{-1} \partial_{x^{i}}$ in the physical frame. For this reason, in our opinion, the measurement of the fermion energy remains an open problem that must be studied carefully before drawing definitive conclusions.

In spite of these difficulties, we hope that the genuine kinematic study presented here may improve the theory of the cosmic neutrinos emitted by remote sources in various epochs of our expanding universe.

Data Availability Statement This manuscript has no associated data or the data will not be deposited. [Authors' comment: This theoretical work does not use or produce numerical data.]

Open Access This article is licensed under a Creative Commons Attribution 4.0 International License, which permits use, sharing, adaptation, distribution and reproduction in any medium or format, as long as you give appropriate credit to the original author(s) and the source, provide a link to the Creative Commons licence, and indicate if changes were made. The images or other third party material in this article are included in the article's Creative Commons licence, unless indicated otherwise in a credit line to the material. If material is not included in the article's Creative Commons licence and your intended use is not permitted by statutory regulation or exceeds the permitted use, you will need to obtain permission directly from the copyright holder. To view a copy of this licence, visit http://creativecomm ons.org/licenses/by/4.0/.

Funded by SCOAP ${ }^{3}$.

\section{Appendix A: Fermions in physical frames}

As the physical frames are less popular, we present few technical details starting with the form of the local covariant derivatives $\hat{\nabla}_{\hat{\alpha}}=\hat{e}_{\hat{\alpha}}^{\mu} \nabla_{\mu}$ that read

$\hat{\nabla}_{0}=\partial_{t}+\frac{\dot{a}(t)}{a(t)} x^{i} \partial_{x^{i}}, \quad \hat{\nabla}^{i}=\partial_{x^{i}}+\frac{1}{2} \frac{\dot{a}(t)}{a(t)} \gamma^{0} \gamma^{i}$.

Furthermore, we give the form of the point-dependent Dirac matrices

$$
\gamma^{0}(x)=\gamma^{0}, \quad \gamma^{i}(x)=\gamma^{i}+x^{i} \frac{\dot{a}(t)}{a(t)} \gamma^{0}
$$

and the covariant derivatives

$$
\nabla_{0}=\partial_{t}-\frac{1}{2} \frac{\dot{a}(t)^{2}}{a(t)^{2}} \gamma^{0} \gamma^{i} \partial_{x^{i}}, \quad \nabla_{i}=\partial_{x^{i}}+\frac{1}{2} \frac{\dot{a}(t)}{a(t)} \gamma^{0} \gamma^{i},
$$

$\nabla^{0}=\partial_{t}+\frac{\dot{a}(t)^{2}}{a(t)^{2}} x^{i} \partial_{x^{i}}$

$\nabla^{i}=-\partial_{x^{i}}-\frac{1}{2} \frac{\dot{a}(t)}{a(t)} \gamma^{0} \gamma^{i}+\frac{\dot{a}(t)^{2}}{a(t)^{2}} x^{i} x^{j} \partial_{x^{j}}$.

in the gauge defined in Eqs. (9) and (10). The Dirac operator [44],

$$
\begin{aligned}
E_{D} & =i \gamma^{\hat{\alpha}} \hat{\nabla}_{\hat{\alpha}}=i \gamma^{\mu}(x) \nabla_{\mu} \\
& =i \gamma^{0} \partial_{t}+i \gamma^{i} \partial_{x^{i}}+i \gamma^{0} \frac{\dot{a}(t)}{a(t)}\left(x^{i} \partial_{x^{i}}+\frac{3}{2}\right),
\end{aligned}
$$

in physical frames has the kinetic part of the flat case completed by the last term giving the coupling to the gravity of the space-times $(M, a)$.

\section{References}

1. C.Y. Cardall, G.M. Fuller, Phys. Rev. D 55, 7960 (1997)

2. N. Fornengo, C. Giunti, C.W. Kim, J. Song, Phys. Rev. D 56, 1895 (1997)

3. D.V. Ahluwalia, C. Burgard, Gen. Relativ. Gravit. 28, 1161 (1996)

4. Y. Grossman, H.J. Lipkin, Phys. Rev. D 55, 2760 (1997)

5. O. Luongo, G.V. Stagno, Mod. Phys. Lett. A 26, 1257 (2011)

6. A. Geralico, O. Luongo, Phys. Lett. A 376, 1239 (2012)

7. L. Buoninfante, G.G. Luciano, L. Petruzziello, L. Smaldone, Phys. Rev. D 101, 024016 (2020)

8. M. Blasone, G. Lambiase, G.G. Luciano, L. Petruzziello, L. Smaldone, Class. Quantum Gravity 37, 155004 (2020)

9. F. Sorge, S. Zilio, Class. Quantum Gravity 24, 2653 (2007)

10. G. Lambiase, G. Papini, R. Punzi, G. Scarpetta, Phys. Rev. D 71, 073011 (2005)

11. G. Lambiase, Eur. Phys. J. C 19, 553 (2001)

12. T. Bhattacharya, S. Habib, E. Mottola, Phys. Rev. D 59, 067301 (1999)

13. R.M. Crocker, C. Giunti, D.J. Mortlock, Phys. Rev. D 69, 063008 (2004)

14. J. Alexandre, K. Clough, Phys. Rev. D 98, 043004 (2018)

15. A. Capolupo, G. Lambiase, A. Quaranta, Phys. Rev. D 101, 095022 (2020)

16. H. Swami, K. Lochan, K.M. Patel, Phys. Rev. D 102, 024043 (2020)

17. S. Nussinov, Phys. Lett. B 63, 201 (1976)

18. S.M. Bilenky, B. Pontecorvo, Phys. Rep. 41, 225 (1978)

19. B. Kayser, Phys. Rev. D 24, 110 (1981)

20. C. Giunti, C.W. Kim, U.W. Lee, Phys. Rev. D 44, 3635 (1991)

21. C. Giunti, C.W. Kim, J.A. Lee, U.W. Lee, Phys. Rev. D 48, 4310 (1993)

22. J. Rich, Phys. Rev. D 48, 4318 (1993)

23. K. Kiers, S. Nussinov, N. Weiss, Phys. Rev. D 53, 537 (1996)

24. W. Grimus, P. Stockinger, Phys. Rev. D 54, 3414 (1996)

25. J.E. Campagne, Phys. Lett. B 400, 135 (1997)

26. W. Grimus, P. Stockinger, S. Mohanty, Phys. Rev. D 59, 013011 (1999)

27. W. Grimus, S. Mohanty, P. Stockinger, Phys. Rev. D 61, 033001 (2000)

28. A.E. Bernardini, S.D. Leo, Phys. Rev. D 70, 053010 (2004) 
29. I.I. Cotăescu, Eur. Phys. J. C 81, 667 (2021)

30. I.I. Cotăescu, Eur. Phys. J. C 81, 908 (2021)

31. P. Painleve, C. R. Acad. Sci. (Paris) 173, 677 (1921)

32. A. Gullstrand, Arkiv. Mat. Astron. Fys. 16, 1 (1922)

33. I.I. Cotăescu, Chin. Phys. C 45, 105101 (2021)

34. G. Lemaître, J. Math. Phys. (Cambridge, Mass.) 4, 188 (1925)

35. I.I. Cotăescu, J. Phys. A Math. Gen. 33, 9177 (2000)

36. S. Drell, J.D. Bjorken, Relativistic Quantum Fields (Me Graw-Hill Book Co., New York, 1965)
37. N.D. Birrel, P.C.W. Davies, Quantum Fields in Curved Space (Cambridge University Press, Cambridge, 1982)

38. W. Rindler, MNRAS 116, 662 (1956)

39. W. Rindler, Essential of Relativity (Springer, Heidelberg, 1969)

40. G.E. Lemaître, Ann. Soc. Sci. de Bruxelles 47A, 49 (1927)

41. G.E. Lemaître, MNRAS 91, 483 (1931)

42. E. Hubble, Proc. Natl. Acad. Sci. 15, 168 (1929)

43. E. Harrison, Astrophys. J. 403, 28 (1993)

44. I.I. Cotaescu, Mod. Phys. Lett. A 22, 2965 (2007) 